\title{
Host genetic variation and its microbiome interactions within the Human Microbiome Project
}

Raivo Kolde ${ }^{1}$, Eric A. Franzosa ${ }^{2,3}$, Gholamali Rahnavard ${ }^{2,3}$, Andrew Brantley Hall ${ }^{3}$, Hera Vlamakis ${ }^{3}$, Christine Stevens ${ }^{3}$, Mark J. Daly ${ }^{3,4}$, Ramnik J. Xavier ${ }^{1,3,5^{*}}$ and Curtis Huttenhower ${ }^{2,3^{*}}$

\begin{abstract}
Background: Despite the increasing recognition that microbial communities within the human body are linked to health, we have an incomplete understanding of the environmental and molecular interactions that shape the composition of these communities. Although host genetic factors play a role in these interactions, these factors have remained relatively unexplored given the requirement for large population-based cohorts in which both genotyping and microbiome characterization have been performed.
\end{abstract}

Methods: We performed whole-genome sequencing of 298 donors from the Human Microbiome Project (HMP) healthy cohort study to accompany existing deep characterization of their microbiomes at various body sites. This analysis yielded an average sequencing depth of 32x, with which we identified 27 million (M) single nucleotide variants and $2.3 \mathrm{M}$ insertions-deletions.

Results: Taxonomic composition and functional potential of the microbiome covaried significantly with genetic principal components in the gastrointestinal tract and oral communities, but not in the nares or vaginal microbiota. Example associations included validation of known associations between FUT2 secretor status, as well as a variant conferring hypolactasia near the LCT gene, with Bifidobacterium longum abundance in stool. The associations of microbial features with both high-level genetic attributes and single variants were specific to particular body sites, highlighting the opportunity to find unique genetic mechanisms controlling microbiome properties in the microbial communities from multiple body sites.

Conclusions: This study adds deep sequencing of host genomes to the body-wide microbiome sequences already extant from the HMP healthy cohort, creating a unique, versatile, and well-controlled reference for future studies seeking to identify host genetic modulators of the microbiome.

Keywords: Human Microbiome Project, Microbiome and human genetics, Human genome sequence, Microbiome metagenome sequence, Association studies

\footnotetext{
* Correspondence: xavier@molbio.mgh.harvard.edu;

chuttenh@hsph.harvard.edu

${ }^{1}$ Center for Computational and Integrative Biology, Massachusetts General

Hospital, 185 Cambridge St, Boston, MA 02114, USA

${ }^{2}$ Department of Biostatistics, Harvard T. H. Chan School of Public Health, 655

Huntington Ave, Boston, MA 02115, USA

Full list of author information is available at the end of the article
} 


\section{Background}

The Human Microbiome Project (HMP) was the first population-scale, body-wide metagenomic microbiome survey, with initial results published in 2012 [1]. Covering 18 clinically relevant body sites from five major body regions in a cohort of 300 healthy adult donors, analysis of HMP data revealed a high degree of microbial community specialization as well as considerable variation in overall microbiome composition between individuals. Providing a baseline of healthy microbial variation, HMP data have served as a versatile reference in numerous studies [2-5]. Examples of how metagenomic data from the HMP have been used include characterizing mobile gene content in the microbiome [6], identifying the prevalence of specific enzymes in human microbiome samples [7, 8], dissecting factors that shape skin microbial communities [9], contrasting ancient and modern oral microbiomes [10], and studying the gut antibiotic resistome [11].

In this study, we provide further data to characterize potential interactions between the microbiome and the human host, specifically, genome sequencing for the HMP cohort. While interpersonal variation in the microbiome can be considerable, the microbiome of specific individuals can conversely be remarkably stable [12-14], suggesting host genetic background as one factor maintaining the composition of microbial communities across the body. Genetic factors influencing microbiome composition have been previously analyzed in mouse models, where external factors such as diet can be tightly controlled. In these studies, host genotype explained a significant proportion of variation in the gut microbiome of intercrossed mouse lines [15-17]. The quantitative trait loci (QTLs) emerging from these studies included genes involved in immune function, for example, Irak3 [15] and Irak4 [17].

The study of genetic effects on the microbiome becomes far more complex in humans. In addition to genetics, microbiome composition is strongly influenced by environmental factors such as diet, overall health status, and medication use $[18,19]$. With the exception of research on special populations like the Hutterites [20], these factors are difficult to constrain in a human study. Nevertheless, some evidence does exist for genetic effects on human microbiome composition. For example, the gut microbiomes of monozygotic twins are significantly more similar than those of dizygotic twins [21, 22]. Recent work has suggested that microbiome heritability is lower than many other traits, however, and not distributed equally among taxa (e.g., with a higher heritability among Firmicutes than Bacteroidetes [22, 23]).

More detailed mapping of associations between single gene variants and microbial taxa has been successful in a targeted approach of candidate genes or variants, where clear molecular mechanisms have been established. For example, inflammatory bowel disease risk loci near NOD2 were also associated with Enterobacteriaceae in patients with inflammatory bowel disease [24]. In addition, variants near the lactase ( $L C T)$ gene, responsible for lactose tolerance, have been associated with abundance of Bifidobacterium [22, 25, 26]. Bifidobacterium has also been associated with a loss-of-function variant in the fucosyltransferase 2 (FUT2) gene, responsible for the transfer of the terminal fucose residues to the mucosal glycans [27].

Despite these examples, very few individual microbepolymorphism associations have been identified that have reached genome-wide significance. Cohort size is certainly a main limiting factor. Studies to date have included up to 1800 individuals, a relatively small number for a successful genome-wide association study of any trait, and especially so considering that the heritability of microbial features can be low relative to many quantitative traits. Furthermore, clinically relevant associations might be discoverable only in cohorts with particular diseases, for example, in conditions such as inflammatory bowel disease or rheumatoid arthritis, which are both genetically complex and accompanied by microbial dysbioses [28, 29].

To facilitate future microbiome-genetic association meta-analyses and to provide a baseline characterization of the HMP population, we report here on wholegenome sequencing from the blood of $298 \mathrm{HMP}$ participants. The data achieve an average of $32 x$ coverage, allowing us to discover two times as many variants as were previously identified using "contaminant" human reads from a subset of 93 whole metagenome sequencing (WMS) samples [25, 30]. The common variants we identified are consistent with findings from other largescale sequencing projects such as 1000 Genomes [31] and Genome of the Netherlands (GoNL) [32]; in addition, we identified numerous novel rare variants in the HMP cohort. In combination with more than 7500 microbiome samples sequenced (including both $16 \mathrm{~S}$ rRNA gene sequencing and WMS) from multiple body sites of the HMP cohort to date, this study creates a unique dataset for studying the microbiome of multiple body sites in the context of host genetics.

\section{Methods}

\section{Genome sequencing}

Genome sequencing was performed at the Broad Institute using polymerase chain reaction (PCR)-free library preparation on Illumina HiSeq $\mathrm{X}$ Ten machines. Reads were mapped to the genome using Burrows-Wheeler Aligner [33], and variants were called with Genome Analysis Toolkit (GATK) version 3.4 on human genome build b37. The sequencing quality was good in all samples according to 
multiple metrics, so no samples were excluded. We used only variants that passed Variant Quality Score Recalibration filtering. Additionally, we filtered out variants in lowcomplexity regions of the genome as defined by $\mathrm{Li}$ et al. [34]. For comparison analyses, we downloaded variant files from the GoNL consortium (https://molgenis26.target.rug.n 1/downloads/gonl_public/variants/release5/) and $1000 \mathrm{Ge}-$ nomes phase 3 (ftp://ftp.1000genomes.ebi.ac.uk/vol1/ftp/rel ease/20130502/) and searched for the presence of all our variants in these other cohorts.

We estimated the impact of coding variants using Variant Effect Predictor (VEP) [35] with Ensembl version 82 together with the Loss-Of-Function Transcript Effect Estimator (LOFTEE) plugin (https://github.com/konr adjk/loftee). To classify mutations into severity groups, we used the annotations provided by VEP. If one variant was located within several transcripts and therefore had multiple potential effects on the coding sequence, we annotated the variant with the most severe outcome.

For principal component analysis (PCA) on the variants, we used 5.9 million $(\mathrm{M})$ common (minor allele frequency $(\mathrm{MAF})>0.05$, call rate $>95 \%$ ) single nucleotide variants (SNVs) in PLINK 1.9 [36]. For joint PCA, we first extracted a similar subset of $6.8 \mathrm{M}$ SNVs from the 1000 Genomes data (MAF $>0.05$, call rate $>95 \%)$, merged the resulting files, and performed PCA using PLINK 1.9.

Kinship analysis was performed using the Kinshipbased INference for Genome-wide association studies (KING) algorithm [37]. The ranges used for inferring degree of relation from kinship coefficients were taken from the original publication and were [0.0442, 0.0884) for third degree, $[0.0884,0.177)$ for second degree, and $[0.177,0.354)$ for first degree relatives, and $>0.354$ for twins. Kinship coefficients below 0.0442 were considered unrelated.

\section{Microbiome data}

All metagenomic data underwent quality control according to the HMP protocol [38, 39]. We performed taxonomic profiling of bacteria, archaea, microbial eukaryotes, and viruses using MetaPhlAn2 [40]. Briefly, MetaPhlAn2 maps shotgun metagenomic sequencing reads against a precomputed database of clade-specific marker genes (i.e., genes that tend to be found in isolate genomes from a given clade, but are rarely seen in isolate genomes outside that clade). Marker gene abundance is averaged withinclade to produce a robust estimate of the clade's genomic coverage in the sample, which can then be normalized to relative abundance units.

We used MetaPhlAn2's taxonomic profiles to guide species-resolved functional profiling with HMP Unified Metabolic Analysis Network 2 (HUMAnN2) (http://huttenh ower.sph.harvard.edu/humann2). For a given metagenome,
HUMAnN2 constructs a sample-specific database by concatenating and indexing the pangenomes of species detected in the sample (species' pangenomes are precomputed, reduced representations of the protein-coding sequences from isolates of a given species [41]). HUMAnN2 then maps sample reads against the corresponding sample-specific database to quantify gene presence and abundance on a per-species basis; reads that fail to map to one of the detected species ("unclassified reads") are separately mapped by translated search against a reduced protein sequence catalog [42]. Finally, HUMAnN2 compares community total, speciesresolved, and unclassified gene family abundance to the MetaCyc pathway catalog [43] to reconstruct metabolic pathway abundance and coverage using the original HUMAnN algorithm [44].

To filter the MetaPhlAn relative abundances for further testing, we used only species-level data and required a species to be present in at least $25 \%$ of the samples in a given body site. This reduced the number of species from 567 to 119 in stool, from 428 to 119 in buccal mucosa, from 479 to 161 in supragingival plaque, from 461 to 156 in tongue dorsum, from 380 to 29 in anterior nares, and from 367 to 23 in posterior fornix. We then applied $\log _{10}$ transformation on their relative abundances (with a pseudocount of $10^{-5}$ ) to stabilize the variation for linear modeling.

Metabolic pathway abundance was much more stable than species-level abundance: for example, more than $78 \%$ of the pathways detected were present in more than $75 \%$ of stool samples. As the number of samples where a pathway was not detected was relatively small for each pathway, we used only the abundances that were present for statistical testing, and used only pathways that were present in more than $75 \%$ of the samples per body site. This reduced the number of species from 756 to 500 in stool, from 706 to 427 in buccal mucosa, from 750 to 511 in supragingival plaque, from 744 to 509 in tongue dorsum, from 742 to 355 in anterior nares, and from 697 to 247 in posterior fornix. Finally, we applied $\log _{10}$ transformation to the read-counts-per-million pathway abundance values returned by the HUMAnN2 pipeline.

In both cases, if multiple samples were collected from the same person and body site, we averaged the relative abundance values. The final number of donors was 209 for stool, 159 for buccal mucosa, 169 for supragingival plaque, 185 for tongue dorsum, 128 for anterior nares, and 80 for posterior fornix.

\section{Genome and microbiome associations}

To associate genomic PCA with the microbiome, we fit a linear model to each of the microbial features, predicting it using the first five principal components. Based on the residuals, we calculated the amount of variance explained $\left(R^{2}\right)$. To put the calculated values into context, 
we shuffled the sample labels 10,000 times and calculated $R^{2}$ for each of the shuffled datasets. The average $R^{2}$ statistic per site was averaged over all features, and the Z-score was calculated based on the same statistic in the 10,000 permuted datasets. For single features the empirical $p$ value was calculated as the proportion of permuted $R^{2}$ scores larger than the actual score.

Starting with ranking of pathways based on their correlation to genomic principal components, we sought to identify classes enriched in the top of the rankings. For this analysis we used the pathway superclass assignments by MetaCyc [43]. For each superclass in the data, we extracted the rankings of its members and calculated a $p$ value [45] showing how strongly the rankings were skewed towards the top.

For genome-wise associations we employed Matrix eQTL software [46] to fit ordinary linear models to predict microbiome features, taking originating site, sex, and ethnicity into account as covariates. Given the set of donors available for each body site, we included only the SNVs with MAF $>0.1$. The variants found in the analysis were associated with genes that were located within $50 \mathrm{~kb}$ of the variant. The variants reported in figures and supplementary tables were filtered as follows: (1) we divided all SNVs associated to a particular species into groups where the distance between consecutive SNVs was not longer than 10,000 bp; (2) from each group of associations, we selected the one with the smallest $p$ value.

\section{Results}

\section{High-quality DNA sequencing of the HMP cohort}

The HMP cohort design and sample collection has been described in depth [47]. Briefly, the HMP cohort comprises 300 donors recruited in two locations in the USA. The majority of donors $(71 \%)$ were of Caucasian origin; the remaining donors were of African (6\%), Asian (9\%),
Latino (11\%), or mixed (3\%) ancestry. The male-tofemale ratio was roughly equal, with 151 females and 149 males. The goal in selecting donors was to find healthy individuals with no recent medication use or disease history, who belonged to a similar age group (19-40 years), and who had a relatively healthy body mass index (BMI of $19-34 \mathrm{~kg} / \mathrm{m}^{2}$ ).

To obtain host genome information, genomic DNA from the blood of 298 of the 300 individuals was sequenced using PCR-free sequencing. The average sequencing coverage was $32.77 x$, with a range of $23.9 x$ to $56.7 \times$ (Fig. 1). Contamination and the percentage of chimeric reads were both well under the standard cutoff of $5 \%$ in all samples (Additional file 1: Figure S1A). The distribution of other quality metrics such as insert size and percentage of reads that aligned in pairs did not highlight any clear outlier samples; therefore, all samples were included in further analysis. The variant number was also remarkably stable at $\sim 2 \mathrm{M}$ single nucleotide polymorphisms (SNPs) and $200 \mathrm{~K}$ indels per person (Fig. 1), with the exception of the African-American donors, who had higher genetic diversity (Additional file 1: Figure S1B). There was no detectable correlation between sequencing depth and number of variants recovered, indicating that depth in all samples was sufficient.

These data provide an almost complete pairing of human genome sequencing to microbial amplicons and metagenomes across the entire HMP cohort. Genetic variation in this cohort was previously inferred using "contaminant" human reads from 93 subjects' WMS data $[25,30]$. While this provided an average human genome coverage of $\sim 10 \times$, it varied greatly between samples and for many reached only $5 \times$. This was sufficient to detect $13 \mathrm{M}$ genetic variants overall, $5.5 \mathrm{M}$ with MAF $>0.05$. In comparison, our study more than tripled the number of donors, and by directly targeting the host

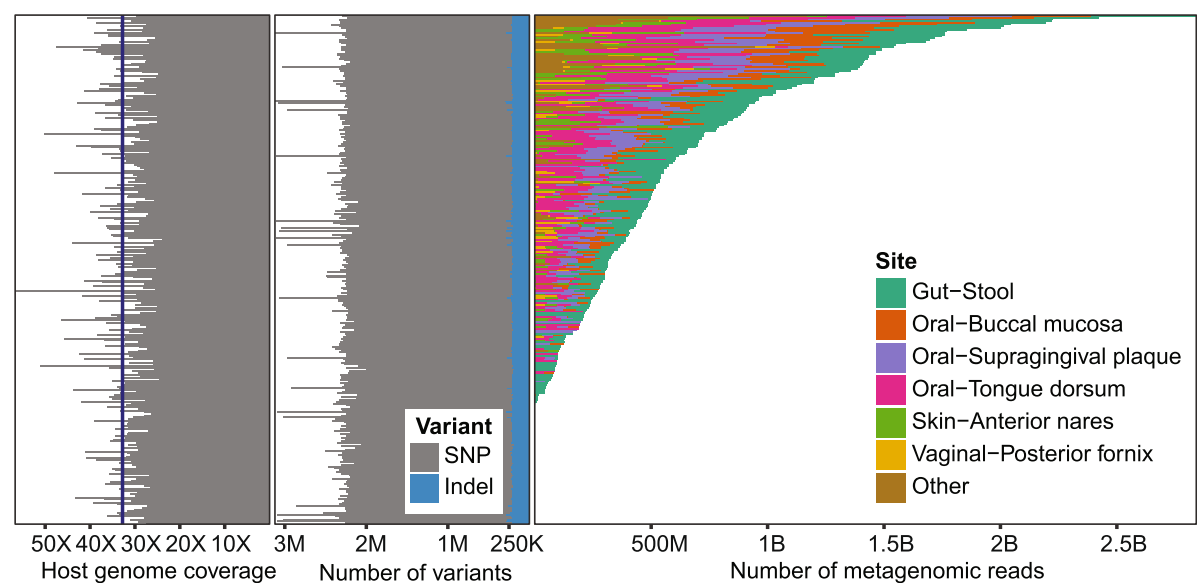

Fig. 1 Overview of the Human Microbiome Project host genome and metagenome coverage. Sequencing depth for each host genome (left) and number of reads for all available samples with whole metagenome sequencing 
DNA, we identified twice as many variants overall with even coverage (minimum $25 \times$ ) between samples. This increased the quality over all samples and generated a complete dataset that can be mined in this work as well as in future studies.

\section{Sequencing results are consistent with those of other comparable populations}

After filtering according to quality and location in lowcomplexity regions, $29 \mathrm{M}$ variants remained, consisting of 26.7 M SNVs and 2.3 M insertions-deletions (indels) (Additional file 1: Table S1). When compared to the GoNL [32] and the 1000 Genomes [31] Projects, 5.1 M SNVs and $856 \mathrm{~K}$ indels were novel, but the majority of these were rare (Fig. 2a). In contrast, the common variants we identified (MAF $>5 \%$ ) were almost universally shared between the three cohorts. Overall, we identified 7.8 M more variants compared to the similarly sized GoNL consortium. Since many of the variants were also present in the 1000 Genomes Project, we attribute the difference to the greater ethnic diversity in the HMP cohort. Although a large number of SNVs were unique to each cohort, the proportion of variants falling in intronic, exonic, and intergenic regions of the genome was almost identical between cohorts (Additional file 1: Figure S2).

We next annotated coding variants using the LOFTEE plugin for the VEP tool [35], which categorizes variants into classes based on their impact on the coding sequence. The number of high-impact variants, defined as those that would result in loss of function of a particular gene, was 2670 (Additional file 1: Table S2); this result is consistent with active negative selection against these variants. Negative selection was also evident from the allele frequency distribution, as the severity of an allele's impact was strongly related to its frequency in the population. For example, high-impact variants were greatly enriched in variants that were observed only once in our dataset (Fig. 2b, AC1). The distribution of coding mutations among genes was also not uniform, with a small number of genes capturing a large number of variants. Thirty genes showed more than five potential high-impact loss-of-function variants, and six genes had more than ten variants. The small number of genes with high-impact coding mutations suggested that this cohort was too small for burden testing to draw correlations between mutation frequency within a gene and microbial features. Instead, we focused our analysis on identifying associations between common variants and microbial taxa or functional potential.

\section{Microbial taxa and functional potential at six body sites}

For the HMP, microbiome samples were collected from 18 body sites, falling into five major areas: gastrointestinal (GI) tract, oral cavity, skin, nares, and vagina. In some cases replicate samples were collected over time to assess temporal stability of the microbiome. In total, more than 5000 samples were characterized using $16 \mathrm{~S}$ rRNA gene sequencing and more than 2000 using shotgun WMS. The former approach gives a high-level overview of taxonomic composition, whereas the latter allows species-level identification and profiling of functional potential of the microbiome. We therefore used WMS data in subsequent analyses. The distribution of samples with WMS was not equivalent between body sites, with most samples drawn from six locations representing four of the major areas described above: gut (stool), oral (buccal mucosa, supragingival plaque, tongue dorsum), nares (anterior nares), and vaginal (posterior fornix); no WMS data were available from the skin
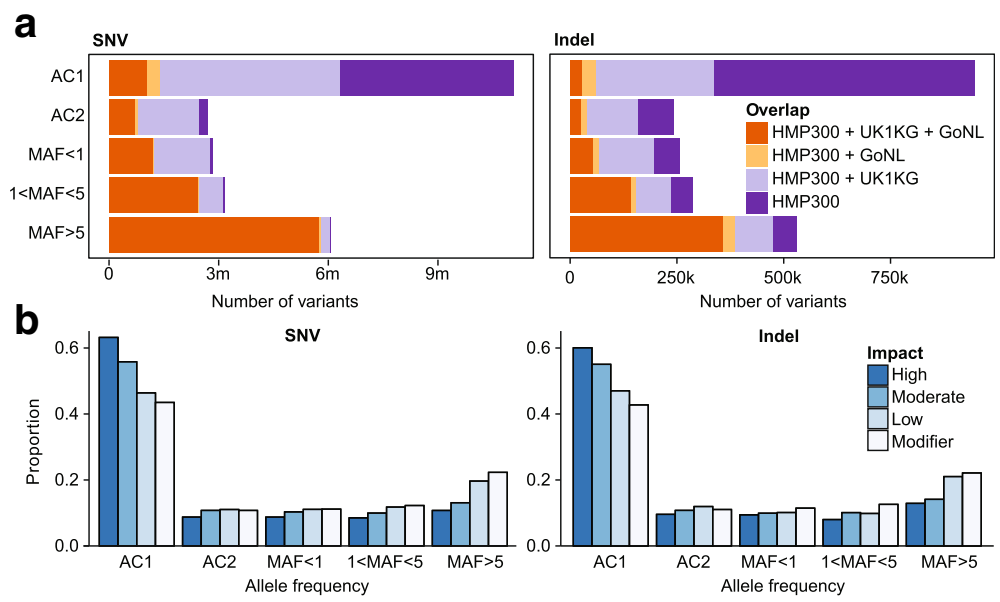

Fig. 2 Distribution of genetic variants and comparison with other cohorts. a Discovered variants categorized by frequency and overlap with other cohorts. AC allele count, MAF minor allele frequency. $\mathbf{b}$ Distribution of the number of coding mutations by frequency and estimated impact 
samples. Within the six body sites, the number of donors ranged from 80 for vaginal posterior fornix to 209 for gut samples; the average number of reads per sample ranged from $34 \mathrm{M}$ in posterior fornix to $86 \mathrm{M}$ in tongue dorsum. Using the WMS data from these samples, we identified taxonomic composition using MetaPhlan2 [40] and functional potential using HUMAnN2 [44]. These outputs were then analyzed for associations with host genetic variation.

\section{Human genomic principal components correlate with microbiome composition}

To compare host genetic variation with microbial variation, we first assessed the degree to which high-level genetic patterns could be correlated with microbiome composition. PCA on the common SNVs (MAF>0.05) demonstrated that the first five principal components predominantly represented the ethnic and racial ancestry of the donors. For example, host genetics of the AfricanAmerican, one of two groups of Asian-American, and the Caucasian subjects showed the strongest effect (Fig. 3a). To further compare overall genetic variation to other cohorts, we also jointly ordinated a combined dataset of HMP300 and 1000 Genomes participants, using SNVs with MAF $>0.05$ in both cohorts. Individuals from both cohorts distributed in the resulting principal component space almost identically according to ancestry (Additional file 1: Figure S3).

Next, for HMP300 we calculated what percentage of microbial variation in the six body sites could be explained $\left(R^{2}\right)$ by the first five host-genome principal components. In stool samples, the percent of specieslevel variation explained by the host principal components was $3.8 \%$, higher than expected by chance alone (empirical $p=0.0001$; Fig. 3b). The distribution of empirical $p$ values for the $R^{2}$ values of the individual species
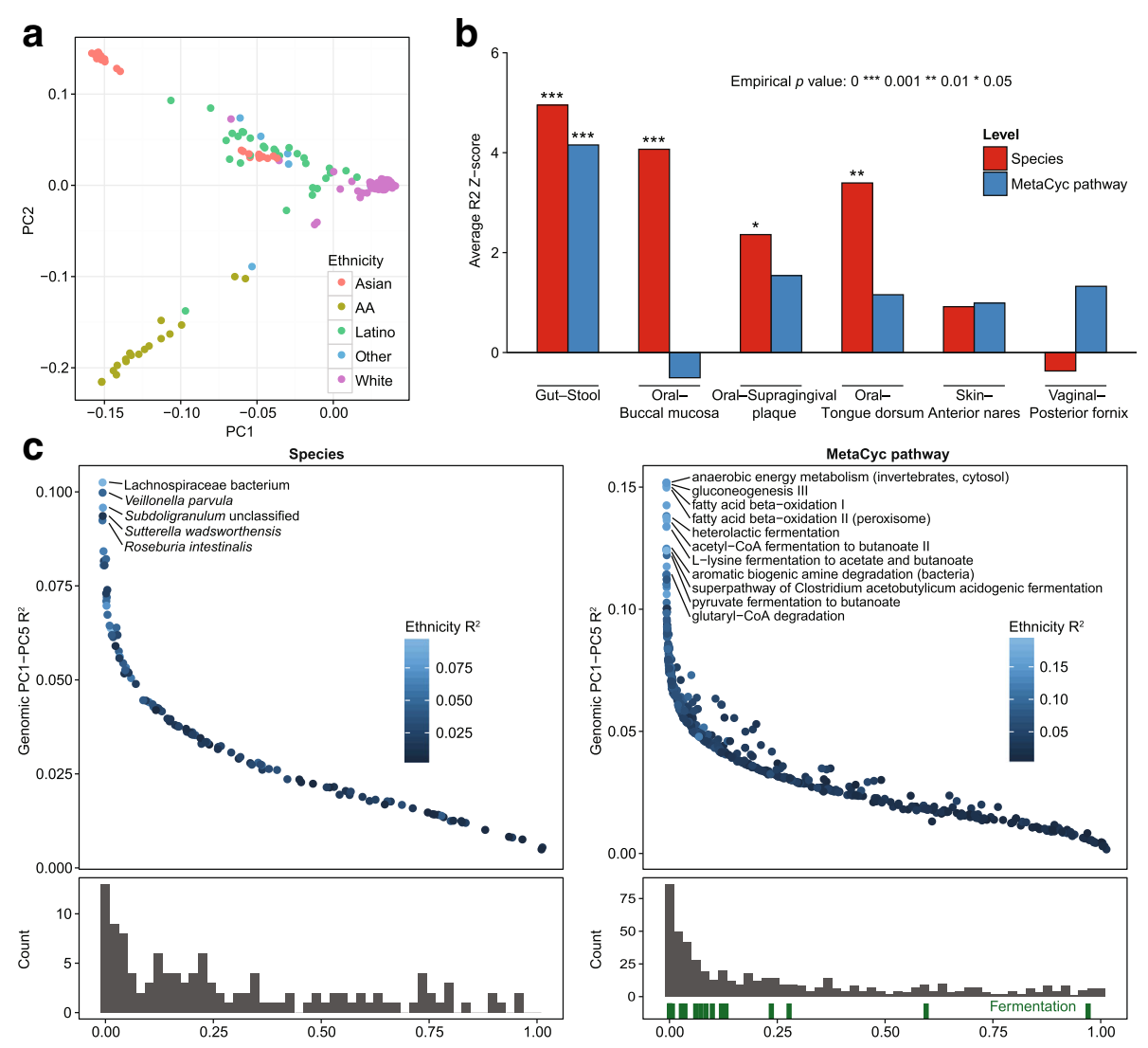

Fig. 3 Correlation between high-level genetic variation and microbiome composition. a The first two components of the genetic principal component analysis are shown, based on common single nucleotide variants, overlaid by self-reported donor ethnicity. AA African-American. b Shown is how much variance in microbiome data on average can be explained by the genetic principal components, when compared to permutation on the same data. Values shown are Z-scores based on permutations, which were also used to calculate empirical $p$ values. $\mathbf{c}$ Distribution of genetic principal component $R^{2}$ values for different species and pathways in stool. Y-axis shows the variance explained, and the $X$-axis shows permutation-based empirical $p$ values for each of those numbers. Only the names of species with false discovery rate (FDR) $<0.05$ and pathways' FDR $<0.01$ are shown. The histogram below displays the distribution of empirical $p$ values, and the $Y$-axis shows the number of species in a bin. Green bars under the pathway histogram show how the pathways that are associated with fermentation are ranked by $R^{2}$ 
was strongly shifted towards zero (Fig. 3c), indicating that strong correlations were not limited to a few species, but that genetic population structure influenced overall microbial configurations. We observed a similar effect on the species level in oral sites. In buccal mucosa, the genomic principal components described on average $5.2 \%$ (empirical $p=0.0008$ ) of the species-level variation; in tongue dorsum, this figure was $4.1 \%$ (empirical $p=$ 0.0034). In an identical analysis of MetaCyc metabolic pathway abundance, we found only the pathways in the gut microbiomes to be significantly correlated with common variant principal components. In summary, the association between high-level host genetic features and microbiome properties was significant at multiple body sites.

When examining the correlation of individual microbial features with host genetics that contributed to these averages, certain features showed much stronger individual associations. In stool, where the genetic correlation was the strongest, five species out of 118 were significantly associated (false discovery rate (FDR) $<0.05$ by permutation test), with $R^{2}$ values reaching almost $10 \%$ (Fig. 3c). Of these five species, Lachnospiraceae bacterium, Roseburia intestinalis, and Subdoligranulum (unclassified) were all positively correlated with the first genomic principal component, demonstrating that these species have higher abundance in donors of Caucasian origin. Another significant species, Sutterella wadsworthensis, was associated with PC4, which separates donors of Asian origin into two groups. Examining other body sites, we found that Porphyromonas catoniae, Propionibacterium propionicum, and unclassified Gemella were significantly associated with host genomic variation in buccal mucosa (Additional file 1: Figure S4 and Table S1).

A similar pathway-level analysis revealed a large number (82 of 541) of pathways significantly (FDR $<0.05$ as above) correlated with genetic principal components in stool (Fig. 3c). Several pathways were related to amino acid and short-chain fatty acid biosynthesis and degradation. In a more systematic view, we found that the members of the fermentation superclass of the MetaCyc database were significantly enriched in the top pathway rankings (Fig. 3c). Most of these pathways were associated with the first genetic principal component that distinguishes white donors from other racial or ethnic ancestries. Such functional enrichments can point to ethnic differences in diet, but also to genetic variability in the ability to metabolize certain nutrients.

In other body sites, pathway-level variability was on average not correlated with genetic principal components, although some individual correlated pathways were found (Additional file 1: Figure S5 and Table S1). For example, a number of pathways in tongue dorsum microbiomes correlated strongly with genetic principal components. Interestingly, almost all of the associated pathways were related to respiration and the tricarboxylic acid (TCA) cycle, indicative of an oxygen gradient and differences in aerobic respiration by oral organisms of the tongue dorsum between donors. The enrichment of the TCA cycle in the oral microbiome and fermentation in the gut microbiome reflect the dominant metabolic features of the corresponding microbiomes and show how these can be affected by host genetics and environmental factors correlated with genetic ancestry.

\section{Related donors have similar microbiomes}

Although the HMP cohort included donors related to each other, this information was not available in the collected metadata. Genomic sequencing of the donors allowed us to infer the extent of relation between all donor pairs and identify up to third degree relatives among them. Using common SNVs (MAF > 0.05) for the analysis, we identified 11 pairs of first degree relatives and one pair of third degree relatives.

We next sought to determine whether the degree of relation was reflected in the similarity of their microbiomes. For this analysis we calculated the Bray-Curtis distance between all donor pairs and divided the pairs into three groups: same ethnicity, different ethnicity, and relatives (Fig. 4a). As could be expected from the PCA, the samples within ethnic groups were on average slightly more similar than samples from different ethnic groups, but microbiome similarity between related donors was more pronounced. With the exception of the gut, in all tested body sites, microbiome community composition between relatives were more similar than between random donor pairs; in anterior nares and buccal mucosa, the effect was also statistically significant by $t$ test between unrelated and related similarity scores. For vaginal samples the effect was also pronounced, but we did not have enough female-female pairs to achieve statistical significance.

\section{FUT2 and LCT genotypes are associated with Bifidobacterium longum}

To study the influence of individual genetic variants on microbiome composition, we began with known associations of FUT2 and LCT. FUT2 is responsible for the transfer of terminal fucose residues to mucosal glycans [48]. Bifidobacteria also use mucus-derived fucose as a carbon source, and abundance and diversity of $B$. longum is significantly lower in non-secretors (individuals with a premature stop codon in FUT2, rs601338) compared to secretors [27].

To determine whether this association could be verified in the HMP cohort, we searched for microbial species correlated with the host fucosyltransferase secretor genotype $(\mathrm{MAF}=0.41)$. We used linear regression to predict the relative abundance of each individual species 


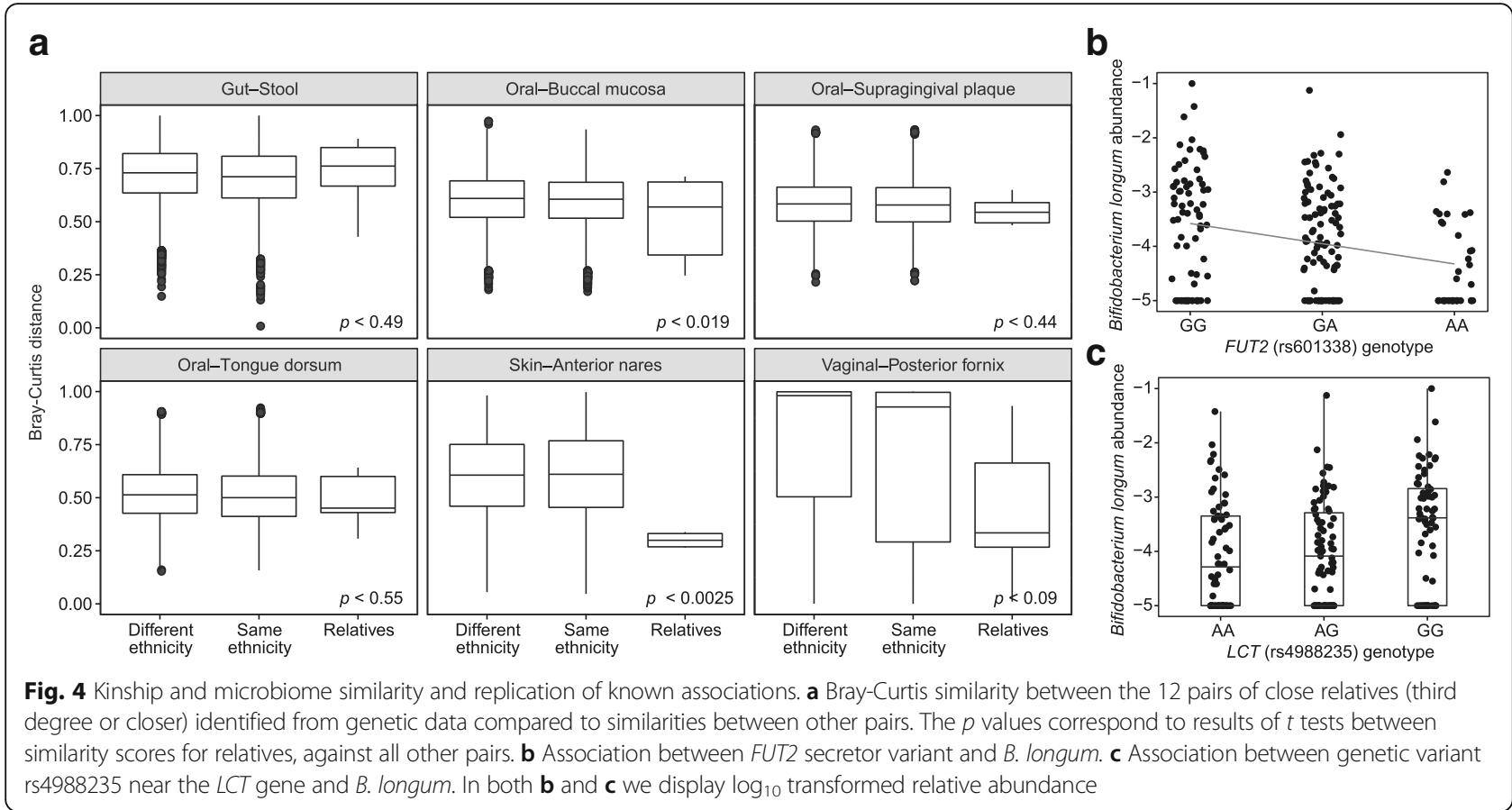

based on the secretor genotype dosage. B. longum had the strongest correlation of the 118 tested species (FDR $=0.018$; Fig. $4 \mathrm{~b}$ ), with increased relative abundance in the secretor genotype relative to the non-secretor genotype. This finding is consistent with previous experimental observations and demonstrates that the cohort is sufficiently powered to validate targeted microbial-host association hypotheses.

Similarly, LCT has been associated with increased abundance of lactose-metabolizing Bifidobacteria in the gut $[22,25]$. LCT encodes lactase, the enzyme responsible for breaking down lactose in the upper GI tract; in tandem with increased Bifidobacteria, this suggests that more dietary lactose collects in the large intestine. The ability to produce lactase in adulthood or lactose intolerance (hypolactasia) is controlled by the presence of a homozygous G allele in rs4988235 SNV close to LCT [49]. A recent finding that milk consumption and Bifidobacteria abundance is positively correlated only in people with the hypolactasia gene variant [26] supports this mechanism.

In the HMP cohort, we compared bacterial species abundances in stool between donors with the hypolactasia and alternative variants. Because hypolactasia is a recessive trait, we used a $t$ test to compare the 64 donors with the homozygous $\mathrm{G}$ genotype to the rest of the 145 donors. After testing each of the 118 individual species abundance against the presence of this variant, we found that $B$. longum had the strongest effect $(\mathrm{FDR}=0.095)$, thereby confirming the previously found association (Fig. 4c).

\section{Microbial associations with host genome variants are body site-specific}

Finally, we assessed associations between host genome and microbiome variation in a non-targeted manner directly through a genome-wide association study. We performed the analysis separately for each body site, concentrating on SNVs with MAF $>0.1$ and comparing them to both microbial species- and metabolic pathwaylevel abundances. We used ordinary linear regression models, taking into account the effects of sex, ethnicity, and sample collection location. After filtering the microbial features (see Methods), we identified 120-160 species in GI tract and oral samples and approximately 25 species in skin and vaginal samples. The number of metabolic pathways passing filtering was considerably higher, between 350 and 530 major pathways per site. Together the large numbers of SNVs, body sites, and microbial features in the analysis impose a strict significance criterion $\left(p<3 \times 10^{-12}\right.$ according to Bonferroni correction for multiple testing), which, in combination with our modest sample size, limits our discovery potential to associations with very large effect sizes.

For this reason, we first limited our analysis to SNVs found in the National Human Genome Research Institute (NHGRI) Genome-Wide Association Studies (GWAS) Catalog [50], hypothesizing that these SNVs were enriched with genomic variants that have potential impact on microbiome properties. This set included SNVs associated with a diverse set of quantitative traits ranging from complex diseases to anthropometric 
measurements. A total of 16,869 of these SNVs were found in our data, but we did not detect any significant associations using this subset of SNVs. Furthermore, according to the quantile-quantile plot of the comparisons, there was no systematic enrichment of smaller $p$ values among the comparisons (Additional file 1: Figures S6 and S7). We did not obtain significant results with even more constrained variant sets associated with inflammatory bowel disease or with any of the high-level GWAS Catalog subcategories (e.g., "immune system disorders," "digestive system").

We next ran the association analysis on all common SNVs. We did not see any associations with $p$ values smaller than multiple testing-corrected significance limits. However, there were a number of associations (Additional file 1: Figures S8 and S9 and Table S2) with relatively small $p$ values. These associations form a rich source of information for future microbiome-wide association studies and investigators interested in specific genes or microbial species.

Because of the unique design of the HMP cohort, we were also able to investigate the nature of the associations between body sites. Studies in expression quantitative trait loci have reported remarkable stability of gene expression-genotype associations across tissues [51]. Here, we did not observe any SNV-microbe pairs in the top of the association rankings for multiple body sites, demonstrating the more indirect nature of these associations as well as the unique community construction principles for each body site. When considering the distribution of the best $p$ values from each body site irrespective of the associated organism, we still could not find any SNVs that were more strongly associated with microbes than expected by chance. This finding further suggests that the genetic mechanisms behind microbiome composition are body site-specific.

\section{Discussion}

Previous studies of the human microbiome have revealed that microbiomes can be remarkably different between individuals, while their composition within an individual remains relatively stable over time. These observations suggest that genetic factors may be one influence on microbiome composition, in addition to recognized ecological and environmental factors such as colonization, diet, medications, and lifestyle. Although studies in twins and mice have suggested some genetic influence on microbiome composition, reports of direct associations are sparse. The main obstacle for such discovery studies is the lack of large well-described cohorts with both microbial abundance and genotype measurements. Here, we report host whole genome sequencing for the HMP cohort, one of the largest and most comprehensively microbially characterized populations in the world.
Host whole-genome sequencing provides the opportunity to associate host genetic variation with microbial features in this cohort. We found that most microbes are correlated to genetic principal components, especially in stool, but also in oral samples. Similar approaches have been applied on subsets of the HMP cohort using haplogroups [30] and common variants [25]. Both of these studies identified associations between high-level genetic features and various microbiome features; however, the mechanistic bases of those associations remain unclear. Some associations are likely to arise from cultural differences in diet or behavior, but human populations also vary in their ability to digest certain nutrients and can thereby create specific microenvironments for gut microbes.

The combination of host and microbiome sequencing in the HMP cohort is unique, inasmuch as it represents the first non-disease population with body-wide metagenomics and deep human whole-genome sequencing. While the $\sim 300$ individuals of the HMP ( 100 with shotgun metagenomics) are of course not enough for a typical association discovery study, the example applications we investigate here are representative of how this data resource might be used in the future. In combination with larger, targeted populations, for example, the HMP cohort can now be used as a baseline, comparator, or validation in microbiome-genetic association studies at most body sites of interest. The high-quality host and microbial data here are appropriate for future metaanalyses and as a methodological framework to make larger discovery efforts more efficient and less costly.

Previous comparisons between monozygotic and dizygotic twins $[22,52]$ have identified a set of microbial taxa with higher than expected heritability coefficients. We examined the behavior of these organisms in our cohort. Unfortunately, not all of the taxa with the highest heritability coefficients were present in our data, likely due to differences in the biases introduced by $16 \mathrm{~S}$ and metagenomic sequencing and subsequent processing. We did apply principal component correlation analysis to the nine heritable taxa that were present in the HMP cohort; however, none were significantly correlated with genetic principal components.

Microbial communities are highly adapted to the environment of particular body locations [1]. We found that associations between microbial and genetic features, on both the host principal component and single-variant levels, were not shared by the microbiomes between body sites. Therefore, association studies within different body sites have the potential to uncover distinct genetic mechanisms influencing biogeographically distinct microbial features. The HMP cohort, with its broad sampling of microorganisms across five major body regions, represents a unique resource for such studies. While the 
number of studies concentrating on the GI tract microbiome is increasing [20, 22-24, 26], the microbial ecologies of other body sites have been studied to a significantly lesser degree.

In our genome-wide association analysis, no individual associations reached the threshold for statistical significance. This is contrast with the findings of Blekhman et al. [25], who reported 83 significant associations using "contaminant" human reads from a subset of HMP participants' metagenomes. The main reason for the discrepancy is the choice of significance thresholds. Blekhman et al. used FDR multiple hypothesis test correction with a threshold of 0.1 , whereas we used a more stringent Bonferroni correction. We felt this to be more appropriate to avoid inflation of FDR values in what is, overall, a fairly small population, particularly due to the numerical properties of the genetic (e.g., linkage disequilibrium) and microbial (e.g., zero inflation and nonnormal distributions) data. Without this assumption and using the FDR $<0.1$ control instead, we see a number of associations across body sites (Additional file 1: Table S2). However, the statistical significance of these associations was not confirmed in subsequent permutation testing.

Nevertheless, many findings between the two datasets were qualitatively similar, including an association between $L C T$ variants and Bifidobacterium abundance that has also been validated in additional external studies $[22,25,26]$. Highly ranked associations from our tests may be similarly relevant findings, such as variants near SULT2B1 that are relatively strongly associated with $A c$ tinomyces viscocus in skin $\left(p=2.4 \times 10^{-8}\right)$. This gene is involved in processing dehydroepiandrosterone, a hormone implicated in epidermal thickness and sebum production of the skin [53]. In addition, the endoplasmic reticulum aminopeptidase 1 (ERAP1) gene, which is involved in antigen presentation and associated with inflammatory bowel disease [54] and a number of other autoimmune diseases [55-57], appeared in two associations. In buccal mucosa samples, ERAP1 was associated with Actinomyces graevenitzii $\left(p=3.9 \times 10^{-8}\right)$, a normal member of the oral microflora that can, under some circumstances, act as an opportunistic pathogen and cause pulmonary abscesses [58]. In the GI tract, different variants close to ERAP1 were associated with Lachnospiraceae bacteria $\left(p=3.2 \times 10^{-8}\right)$. Interestingly, Lachnospiraceae was the most differentially regulated microbial family in the terminal ileum of patients with ankylosing spondylitis [59], a disease associated with polymorphisms in ERAP1 [60]. These and other putative associations present compelling evidence, but will have to be confirmed by future studies.

In general, to reliably identify genetic influences on microbial features, it will be necessary to increase the number of samples with both host and microbial sequencing. This is particularly true when combining baseline "healthy" and disease-specific populations, genetic variants, and microbial variants. For example, based on the NOD2-Enterobacteriaceae association found in patients with inflammatory bowel disease, it was calculated that detecting the same association in a genome-wide significant manner would require $3700 \mathrm{pa}$ tients [24]. In this regard, two recent papers [23, 26] emphasize how good validation cohorts can help to filter results from otherwise underpowered studies.

Combining HMP data with data from other cohorts through meta-analysis or as a validation cohort is thus one way to facilitate future studies. By validating two previous genotype-microbe associations (FUT2 and $L C T$ ), we showed that the HMP genetic data provide power to validate targeted hypotheses and contribute evidence to meta-analyses. Given that the HMP features broad biogeographic microbial sampling across the body, and the human sequencing data are of high quality and consistent with data from projects such as GoNL and 1000 Genomes, these data are well suited for such purposes. The comprehensive nature of whole-genome sequencing makes the data versatile, allowing the combination of HMP data with genome analysis technologies from genotyping arrays for common variants to exome sequencing for coding variation or other targeted approaches.

\section{Conclusions}

Here, we present the results of whole-genome sequencing of donors from the HMP healthy cohort study, enabling the study of host genetic effects on the microbiome properties of multiple body sites in this cohort. We detected significant correlations between high-level genetic features and both microbial species and community functional profiles. Using these data, we verified that variants near the $L C T$ and FUT2 genes associated significantly with the abundance of B. longum in stool. In a broader, untargeted genome-wide setting, we did not identify significant associations with single variants, mainly due to stringent multiple testing criteria imposed by the numbers of microbial features and body sites in combination with a relatively modest sample size. The top associations, however, provide an initial picture of bodywide host-microbial interaction potential. In addition, the dataset as a whole, when paired with the comprehensive microbiome sequencing already performed on this cohort, constitutes an invaluable resource for further studies.

\section{Additional file}

Additional file 1: Figure S1. Sequencing and variant call quality metrics. A. Percentage of contamination and chimeric reads. B. Various metrics based on variant calling. Figure S2. Distribution of genic and intergenic variants. Figure S3. Combined PCA between 1000 Genomes 
and HMP300. Figure S4. Correlation between high-level genetic features and microbial species in non-gut body sites. Figure $\mathbf{S 5}$. Correlation between high-level genetic features and microbial metabolic pathways in non-gut body sites. Figure S6. Quantile-quantile plots for association analysis between microbial species and GWAS Catalog SNVs. Figure S7. Quantile-quantile plots for association analysis between microbial metabolic pathways and GWAS Catalog SNVs. Figure S8. Putative SNV-microbial species associations. Figure S9. Putative SNV-microbial metabolic pathway associations. Table S1. Raw statistics for genetic principal component analysis. Table S2. Top SNV and microbiome association results. (PDF $1953 \mathrm{~kb}$ )

\section{Abbreviations}

AC: Allele count; FDR: False discovery rate; GI: Gastrointestinal; GoNL: Genome of the Netherlands; GWAS: Genome-Wide Association Study; HMP: Human Microbiome Project; LoF: Loss of function; MAF: Minor allele frequency; NHGRI: National Human Genome Research Institute; PCA: Principal component analysis; SNV: Single nucleotide variant; VEP: Variant Effect Predictor; WMS: Whole metagenome sequencing

\section{Acknowledgements}

We thank Andrea Ganna and Manuel Rivas for discussions regarding analysis of genetic variation, Tiffany Poon for handling data submission, and Natalia Nedelsky and Theresa Reimels for editorial assistance and help with figure generation.

\section{Funding}

Funding was provided by the National Institutes of Health $(\mathrm{NIH})$, grants U54HG003067 and NIH U54DE023798, and by the Army Research Office (ARO), grant W911NF-11-1-0429.

\section{Availability of data and materials}

Human genome sequencing data are available from the Database of Genotypes and Phenotypes (dbGaP; http://www.ncbi.nlm.nih.gov/gap) with accession number [dbGaP:phs000228.v4.p1]. Metagenomic sequencing data are available from the Sequence Read Archive (SRA; https:// www.ncbi.n/m.nih.gov/sra) with BioProject numbers PRJNA48479 and PRJNA275349. Precomputed metagenomic profiles and basic subject metadata are available through the HMP Data Analysis and Coordination Center (HMP DACC; http://hmpdacc.org), as described in [39]. Additional subject metadata are available from dbGaP with accession number [dbGaP:phs000228.v3.p1]. Subject metadata, genomic sequencing, metagenomic sequencing, and metagenomic profiles can be linked by HMP-assigned random subject identifiers (field "RANDSID").

\section{Authors' contributions}

MJD, CH, and RJX conceived and designed the study; CS collected experimental data; RK, EAF, and GR performed the computational analysis; $\mathrm{RK}, \mathrm{ABH}, \mathrm{HV}, \mathrm{CH}$, and RJX interpreted the data and drafted the manuscript. All authors read and approved the final manuscript.

\section{Ethics approval and consent to participate}

Recruitment protocols were approved by the appropriate institutional review boards (IRBs) at each HMP clinical site (Baylor College of Medicine, IRB protocols H-22895 (IRB 00001021) and H-22035 (IRB 00002649)); Washington University School of Medicine (IRB protocol HMP-07-001 (IRB 201105198)); and St. Louis University (IRB 15778). Written informed consent was obtained from all study participants to participate in the study and to allow data sharing through $\mathrm{dbGaP}$. All participants consented for the sequencing of their own genetic material [47]. Research on human subjects was performed in accordance with the Declaration of Helsinki. The study was also reviewed by the J. Craig Venter Institute under IRB protocol 2008-084 (IRB 00003721). The study was determined to be exempt from IRB review at the Broad Institute.

\section{Consent for publication}

Not applicable.

\section{Competing interests}

The authors declare that they have no competing interests.

\section{Publisher's Note}

Springer Nature remains neutral with regard to jurisdictional claims in published maps and institutional affiliations.

\section{Author details}

${ }^{1}$ Center for Computational and Integrative Biology, Massachusetts General Hospital, 185 Cambridge St, Boston, MA 02114, USA. ²Department of Biostatistics, Harvard T. H. Chan School of Public Health, 655 Huntington Ave, Boston, MA 02115, USA. ${ }^{3}$ The Broad Institute of MIT and Harvard, 415 Main St, Cambridge, MA 02142, USA. ${ }^{4}$ Center for Human Genetic Research, Massachusetts General Hospital, 185 Cambridge St, Boston, MA 02114, USA ${ }^{5}$ Center for Microbiome Informatics \& Therapeutics, Massachusetts Institute of Technology, Cambridge, MA 02139, USA.

Received: 17 November 2017 Accepted: 4 January 2018

Published online: 29 January 2018

\section{References}

1. Human Microbiome Project C. Structure, function and diversity of the healthy human microbiome. Nature. 2012;486:207-14.

2. Pham TA, Clare S, Goulding D, Arasteh JM, Stares MD, Browne HP, Keane JA, Page AJ, Kumasaka N, Kane L, et al. Epithelial IL-22RA1-mediated fucosylation promotes intestinal colonization resistance to an opportunistic pathogen. Cell Host Microbe. 2014;16:504-16.

3. Aujoulat F, Bouvet $P$, Jumas-Bilak $E$, Jean-Pierre $H$, Marchandin $H$. Veillonella seminalis sp. nov., a novel anaerobic Gram-stain-negative coccus from human clinical samples, and emended description of the genus Veillonella. Int J Syst Evol Microbiol. 2014;64:3526-31.

4. Rodrigues NF, Kastle J, Coutinho TJ, Amorim AT, Campos GB, Santos VM, Marques LM, Timenetsky J, de Farias ST. Qualitative analysis of the vaginal microbiota of healthy cattle and cattle with genital-tract disease. Genet Mol Res. 2015;14:6518-28.

5. Kevans D, Turpin W, Madsen K, Meddings J, Shestopaloff K, Xu W, MorenoHagelsieb G, Griffiths A, Silverberg MS, Paterson A, et al. Determinants of intestinal permeability in healthy first-degree relatives of individuals with Crohn's disease. Inflamm Bowel Dis. 2015;21:879-87.

6. Brito IL, Yilmaz S, Huang K, Xu L, Jupiter SD, Jenkins AP, Naisilisili W, Tamminen M, Smillie CS, Wortman JR, et al. Mobile genes in the human microbiome are structured from global to individual scales. Nature. 2016; 535:435-9.

7. Mao B, Li D, Zhao J, Liu X, Gu Z, Chen YQ, Zhang H, Chen W. In vitro fermentation of fructooligosaccharides with human gut bacteria. Food Funct. 2015;6:947-54.

8. Williams BB, Van Benschoten AH, Cimermancic P, Donia MS, Zimmermann M, Taketani M, Ishihara A, Kashyap PC, Fraser JS, Fischbach MA. Discovery and characterization of gut microbiota decarboxylases that can produce the neurotransmitter tryptamine. Cell Host Microbe. 2014;16:495-503.

9. Oh J, Byrd AL, Deming C, Conlan S, Program NCS, Kong HH, Segre JA. Biogeography and individuality shape function in the human skin metagenome. Nature. 2014;514:59-64.

10. Warinner C, Rodrigues JF, Vyas R, Trachsel C, Shved N, Grossmann J, Radini A, Hancock Y, Tito RY, Fiddyment S, et al. Pathogens and host immunity in the ancient human oral cavity. Nat Genet. 2014;46:336-44.

11. Forslund K, Sunagawa S, Kultima JR, Mende DR, Arumugam M, Typas A, Bork P. Country-specific antibiotic use practices impact the human gut resistome. Genome Res. 2013;23:1163-9.

12. Oh J, Byrd AL, Park M, Program NCS, Kong HH, Segre JA. Temporal stability of the human skin microbiome. Cell. 2016;165:854-66.

13. David LA, Materna AC, Friedman J, Campos-Baptista MI, Blackburn MC, Perrotta A, Erdman SE, Alm EJ. Host lifestyle affects human microbiota on daily timescales. Genome Biol. 2014;15:R89.

14. Ding T, Schloss PD. Dynamics and associations of microbial community types across the human body. Nature. 2014;509:357-60.

15. Benson AK, Kelly SA, Legge R, Ma F, Low SJ, Kim J, Zhang M, Oh PL, Nehrenberg D, Hua $\mathrm{K}$, et al. Individuality in gut microbiota composition is a complex polygenic trait shaped by multiple environmental and host genetic factors. Proc Natl Acad Sci U S A. 2010;107:18933-8.

16. Leamy LJ, Kelly SA, Nietfeldt J, Legge RM, Ma F, Hua K, Sinha R, Peterson DA, Walter J, Benson AK, Pomp D. Host genetics and diet, but not immunoglobulin A expression, converge to shape compositional features of 
the gut microbiome in an advanced intercross population of mice. Genome Biol. 2014;15:552.

17. Org E, Parks BW, Joo JW, Emert B, Schwartzman W, Kang EY, Mehrabian M, Pan C, Knight R, Gunsalus R, et al. Genetic and environmental control of host-gut microbiota interactions. Genome Res. 2015;25:1558-69.

18. Falony G, Joossens M, Vieira-Silva S, Wang J, Darzi Y, Faust K, Kurilshikov A, Bonder MJ, Valles-Colomer M, Vandeputte D, et al. Population-level analysis of gut microbiome variation. Science. 2016;352:560-4.

19. Zhernakova A, Kurilshikov A, Bonder MJ, Tigchelaar EF, Schirmer M, Vatanen T, Mujagic Z, Vila AV, Falony G, Vieira-Silva S, et al. Population-based metagenomics analysis reveals markers for gut microbiome composition and diversity. Science. 2016;352:565-9.

20. Davenport ER, Cusanovich DA, Michelini K, Barreiro LB, Ober C, Gilad Y. Genome-wide association studies of the human gut microbiota. PLoS One. 2015;10:e0140301.

21. Hansen EE, Lozupone CA, Rey FE, Wu M, Guruge JL, Narra A, Goodfellow J, Zaneveld JR, McDonald DT, Goodrich JA, et al. Pan-genome of the dominant human gut-associated archaeon, Methanobrevibacter smithii, studied in twins. Proc Natl Acad Sci U S A. 2011;108 Suppl 1:4599-606.

22. Goodrich JK, Davenport ER, Beaumont M, Jackson MA, Knight R, Ober C, Spector TD, Bell JT, Clark AG, Ley RE. Genetic determinants of the gut microbiome in UK twins. Cell Host Microbe. 2016;19:731-43.

23. Turpin W, Espin-Garcia O, Xu W, Silverberg MS, Kevans D, Smith MI, Guttman DS, Griffiths A, Panaccione R, Otley A, et al. Association of host genome with intestinal microbial composition in a large healthy cohort. Nat Genet. 2016;48(11):1413-7.

24. Knights D, Silverberg MS, Weersma RK, Gevers D, Dijkstra G, Huang H, Tyler $A D$, van Sommeren S, Imhann F, Stempak JM, et al. Complex host genetics influence the microbiome in inflammatory bowel disease. Genome Med. 2014;6:107.

25. Blekhman R, Goodrich JK, Huang K, Sun Q, Bukowski R, Bell JT, Spector TD, Keinan A, Ley RE, Gevers D, Clark AG. Host genetic variation impacts microbiome composition across human body sites. Genome Biol. 2015;16:191.

26. Bonder MJ, Kurilshikov A, Tigchelaar EF, Mujagic Z, Imhann F, Vila AV Deelen $P$, Vatanen T, Schirmer M, Smeekens SP, et al. The effect of host genetics on the gut microbiome. Nat Genet. 2016:48(11):1407-12.

27. Wacklin P, Makivuokko H, Alakulppi N, Nikkila J, Tenkanen H, Rabina J, Partanen J, Aranko K, Matto J. Secretor genotype (FUT2 gene) is strongly associated with the composition of Bifidobacteria in the human intestine. PLoS One. 2011;6:e20113.

28. Zhang X, Zhang D, Jia H, Feng Q, Wang D, Liang D, Wu X, Li J, Tang L, Li Y, et al. The oral and gut microbiomes are perturbed in rheumatoid arthritis and partly normalized after treatment. Nat Med. 2015;21:895-905.

29. Gevers D, Kugathasan S, Denson LA, Vazquez-Baeza Y, Van Treuren W, Ren B, Schwager E, Knights D, Song SJ, Yassour M, et al. The treatmentnaive microbiome in new-onset Crohn's disease. Cell Host Microbe. 2014;15:382-92

30. Ma J, Coarfa C, Qin X, Bonnen PE, Milosavljevic A, Versalovic J, Aagaard K. mtDNA haplogroup and single nucleotide polymorphisms structure human microbiome communities. BMC Genomics. 2014;15:257.

31. Genomes Project C, Auton A, Brooks LD, Durbin RM, Garrison EP, Kang HM, Korbel JO, Marchini JL, McCarthy S, McVean GA, Abecasis GR. A global reference for human genetic variation. Nature. 2015;526:68-74.

32. Genome of the Netherlands Consortium. Whole-genome sequence variation, population structure and demographic history of the Dutch population. Nat Genet. 2014;46:818-25.

33. Li H, Durbin R. Fast and accurate short read alignment with BurrowsWheeler transform. Bioinformatics. 2009;25:1754-60.

34. Li H. Toward better understanding of artifacts in variant calling from highcoverage samples. Bioinformatics. 2014;30:2843-51.

35. McLaren W, Pritchard B, Rios D, Chen Y, Flicek P, Cunningham F. Deriving the consequences of genomic variants with the Ensembl API and SNP Effect Predictor. Bioinformatics. 2010;26:2069-70.

36. Chang CC, Chow CC, Tellier LC, Vattikuti S, Purcell SM, Lee JJ. Secondgeneration PLINK: rising to the challenge of larger and richer datasets. Gigascience. 2015;4:7.

37. Manichaikul A, Mychaleckyj JC, Rich SS, Daly K, Sale M, Chen WM. Robust relationship inference in genome-wide association studies. Bioinformatics. 2010;26:2867-73.

38. Gevers D, Pop M, Schloss PD, Huttenhower C. Bioinformatics for the Human Microbiome Project. PLoS Comput Biol. 2012;8:e1002779.
39. Lloyd-Price J, Mahurkar A, Rahnavard G, Crabtree J, Orvis J, Hall AB, Brady A, Creasy HH, McCracken C, Giglio MG, et al. Strains, functions and dynamics in the expanded Human Microbiome Project. Nature. 2017:550(7674):61-6.

40. Truong DT, Franzosa EA, Tickle TL, Scholz M, Weingart G, Pasolli E, Tett A, Huttenhower C, Segata N. MetaPhIAn2 for enhanced metagenomic taxonomic profiling. Nat Methods. 2015;12:902-3.

41. Huang K, Brady A, Mahurkar A, White O, Gevers D, Huttenhower C, Segata N. MetaRef: a pan-genomic database for comparative and community microbial genomics. Nucleic Acids Res. 2014;42:D617-624.

42. Suzek BE, Wang Y, Huang H, McGarvey PB, Wu CH, UniProt Consortium UniRef clusters: a comprehensive and scalable alternative for improving sequence similarity searches. Bioinformatics. 2015;31:926-32.

43. Caspi $R$, Billington $R$, Ferrer L, Foerster $H$, Fulcher CA, Keseler IM, Kothar A, Krummenacker M, Latendresse M, Mueller LA, et al. The MetaCyc database of metabolic pathways and enzymes and the BioCyc collection of pathway/genome databases. Nucleic Acids Res. 2016;44: D471-480.

44. Abubucker S, Segata N, Goll J, Schubert AM, Izard J, Cantarel BL, RodriguezMueller B, Zucker J, Thiagarajan M, Henrissat B, et al. Metabolic reconstruction for metagenomic data and its application to the human microbiome. PLoS Comput Biol. 2012;8:e1002358.

45. Kolde R, Laur S, Adler P, Vilo J. Robust rank aggregation for gene list integration and meta-analysis. Bioinformatics. 2012;28:573-80.

46. Shabalin AA. Matrix eQTL: ultra fast eQTL analysis via large matrix operations. Bioinformatics. 2012;28:1353-8.

47. Human Microbiome Project C. A framework for human microbiome research. Nature. 2012:486:215-21.

48. Kelly RJ, Rouquier S, Giorgi D, Lennon GG, Lowe JB. Sequence and expression of a candidate for the human Secretor blood group alpha(1,2)fucosyltransferase gene (FUT2). Homozygosity for an enzymeinactivating nonsense mutation commonly correlates with the non-secretor phenotype. J Biol Chem. 1995;270:4640-9.

49. Enattah NS, Sahi T, Savilahti E, Terwilliger JD, Peltonen L, Jarvela I. Identification of a variant associated with adult-type hypolactasia. Nat Genet. 2002:30:233-7.

50. Welter D, MacArthur J, Morales J, Burdett T, Hall P, Junkins H, Klemm A, Flicek P, Manolio T, Hindorff L, Parkinson H. The NHGRI GWAS Catalog, a curated resource of SNP-trait associations. Nucleic Acids Res. 2014;42: D1001-1006.

51. Consortium GT. Human genomics. The Genotype-Tissue Expression (GTEx) pilot analysis: multitissue gene regulation in humans. Science. 2015;348:648-60.

52. Goodrich JK, Waters JL, Poole AC, Sutter JL, Koren O, Blekhman R, Beaumont M, Van Treuren W, Knight R, Bell JT, et al. Human genetics shape the gut microbiome. Cell. 2014;159:789-99.

53. Baulieu EE, Thomas G, Legrain S, Lahlou N, Roger M, Debuire B, Faucounau V, Girard L, Hervy MP, Latour F, et al. Dehydroepiandrosterone (DHEA), DHEA sulfate, and aging: contribution of the DHEAge Study to a sociobiomedical issue. Proc Natl Acad Sci U S A. 2000;97:4279-84.

54. Jostins L, Ripke S, Weersma RK, Duerr RH, McGovern DP, Hui KY, Lee JC, Schumm LP, Sharma $Y$, Anderson CA, et al. Host-microbe interactions have shaped the genetic architecture of inflammatory bowel disease. Nature. 2012;491:119-24.

55. Kirino $Y$, Bertsias $G$, Ishigatsubo $Y$, Mizuki N, Tugal-Tutkun I, Seyahi E, Ozyazgan Y, Sacli FS, Erer B, Inoko H, et al. Genome-wide association analysis identifies new susceptibility loci for Behcet's disease and epistasis between HLA-B*51 and ERAP1. Nat Genet. 2013;45:202-7.

56. Genetic Analysis of Psoriasis Consortium, the Wellcome Trust Case Control Consortium, Strange A, Capon F, Spencer CC, Knight J, Weale ME, Allen MH, Barton A, Band G, et al. A genome-wide association study identifies new psoriasis susceptibility loci and an interaction between HLA-C and ERAP1. Nat Genet. 2010;42:985-90.

57. Li YR, Li J, Zhao SD, Bradfield JP, Mentch FD, Maggadottir SM, Hou C, Abrams DJ, Chang D, Gao F, et al. Meta-analysis of shared genetic architecture across ten pediatric autoimmune diseases. Nat Med. 2015;21: 1018-27.

58. Nagaoka K, Izumikawa K, Yamamoto Y, Yanagihara K, Ohkusu K, Kohno S. Multiple lung abscesses caused by Actinomyces graevenitzii mimicking acute pulmonary coccidioidomycosis. J Clin Microbiol. 2012; 50:3125-8. 
59. Costello ME, Ciccia F, Willner D, Warrington N, Robinson PC, Gardiner B, Marshall M, Kenna TJ, Triolo G, Brown MA. Intestinal dysbiosis in ankylosing spondylitis. Arthritis Rheumatol. 2015;67:686-91.

60. Evans DM, Spencer CC, Pointon JJ, Su Z, Harvey D, Kochan G, Oppermann $\mathrm{U}$, Dilthey A, Pirinen M, Stone MA, et al. Interaction between ERAP1 and HLA-B27 in ankylosing spondylitis implicates peptide handling in the mechanism for HLA-B27 in disease susceptibility. Nat Genet. 2011;43:761-7.

Submit your next manuscript to BioMed Central and we will help you at every step:

- We accept pre-submission inquiries

- Our selector tool helps you to find the most relevant journal

- We provide round the clock customer support

- Convenient online submission

- Thorough peer review

- Inclusion in PubMed and all major indexing services

- Maximum visibility for your research

Submit your manuscript at www.biomedcentral.com/submit
Biomed Central 